\title{
Quadrotor attitude estimation using Adaptive Fading Multiplicative EKF*
}

\author{
Tor-Aleksander Johansen and Raymond Kristiansen
}

\begin{abstract}
This paper presents the implementation of an adaptive fading multiplicative extended Kalman filter (AFMEKF), applied to the problem of attitude estimation in the context of quadrotors. The extended Kalman filter is adapted for use with quaternions and made adaptive to account for inaccurate measurement information. Simulations validating the filters performance.
\end{abstract}

\section{KEY WORDS}

Attitude Estimation, AFEKF (Adaptive Fading Extended Kalman Filter), MEKF (Multiplicative Extended Kalman Filter), Quadrotor, Sensor fusion, Accelerated Flight

\section{INTRODUCTION}

Aerial robotics has become one of the core fields in mobile robotic research [1]. The most popular platform utilized within this area of interest is the quadrotor. Often appreciated for its high maneuverability, low cost, and simplicity the platform consist of four rotor-motor assemblies attached to a rigid cross-frame. The quadrotor platform has been subjected to a range of applications over the last decade. Emerging new categories of application are operations in near-Earth environments such as close to structures, inside tunnels and forests [1]. One example of such an application is disaster relief inside collapsed structures, a type of operation that may require aerobatic autonomous flight.

Making these platforms fully autonomous in such environments requires high quality state estimates. States such as quadrotor position, velocity, attitude and angular velocity are all required for successful autonomous operations [2]. Quadrotors are commonly equipped with a proprioceptive sensor suite consisting of some global positioning system (GPS) and an inertial measurement unit (IMU). Additionally, for autonomous operations, the need for exteroceptive sensors such as vision systems, laser range finders and acoustic sensors quickly arise, however at a cost of more demanding computational requirements. Moreover, in the aforementioned near-Earth environments, measurements from the GPS system is often rendered unsatisfactory or simply unavailable. Our work focuses on the challenge of acquiring accurate high quality attitude estimates during

\footnotetext{
*This research has been funded by the Norwegian Research Council and is part of the Arctic Earth Observation and Surveillance Technologies project 195142/I60.

Both authors are with the Department of Electrical Engineering, UiT The Arctic University of Norway, 8505 Narvik, Norway \{tor-aleksander.johansen, raymond.kristiansen\}@uit.no
}

aerobatic operations in GPS restricted environments without the use of exteroceptive sensors.

Previous work on attitude estimation often involve the application of some sensor fusion algorithm, which in the general sense can be divided into two processes [3]; first estimation of vehicle attitude from measurements, and secondly filtering of the noisy measurements by the application of system models. The most famous of such algorithms is the Kalman filter [4], which provides the optimal estimate minimizing the estimation error covariance for a linear system. The extended Kalman filter (EKF) is a further extension of the Kalman filter [5], [6], applying it to a first-order approximation of the underlying nonlinear system and evaluating the approximation at the some state estimate [7]. As a further development the multiplicative EKF (MEKF) was introduced [8], allowing for multiplication to be used in the measurement update. In [9] the authors derive an adaptive fading EKF (AFEKF) for a fault-tolerant INS-GPS loosely coupled system, where the filter calculates an innovation measure, affected by unaccounted errors and unmodeled dynamics, which then is used to improve the estimate.

Recent advances in the field of quadrotor attitude estimation has seen the integration of the dynamic model of the quadrotor into the estimators. In [10] it is shown that, due to different aerodynamic effects including blade flapping, there exists body-frame horizontal forces that are proportional to the translational body frame velocity. In [?], this fact is exploited in the construction of a novel state estimator for quadrotors, utilizing an enhanced dynamic model of the quadrotor inside the EKF framework, improved attitude estimates along with drift free lateral and longitudinal velocities is obtained. Similarly, in [11], a MEKF using quadrotor dynamics is applied in combination with a graph simultaneous localization and mapping algorithm to estimate attitude and position. The authors of [12] provide efforts on quantifying the improved state estimation that is achieved using the dynamics equations in the state estimation scheme, and their results suggest improved attitude estimates due to the increased information available.

As shown in [13], accelerometers onboard quadrotors do not explicitly measure the gravity vector. This is in contrast to the common assumption of weak acceleration, i.e. the gravity vector is approximated as the accelerometer measurement. In flights near hover this assumption holds quite well, however as it is noted in [14]; "...the accuracy of the estimated attitude, provided by these methods, is far from satisfying when the vehicle is subjected to important accelerations". Methods exist that estimate attitude during aggressive maneuvering, 
most of which rely on complementary GPS measurements of the linear velocity to improve the attitude estimate [15]. Notable exemptions can be found as the alternative method described in [16], in which the authors present a method utilizing a large number of accelerometers mounted in a special configuration to measure the angular acceleration and improve the attitude estimates.

In this paper, we focus on acquiring accurate attitude estimates during accelerated flight using only IMU measurements. Taking inspiration from the AFEKF presented in [9] and combining it with the MEKF framework we describe the design, implementation, and verification of an adaptive fading multiplicative extended Kalman filter (AFMEKF) for real-time attitude estimation on a quadrotor. The main contribution of this paper is the implementation of an adaptive strategy in dealing with accelerated flight. This paper is organized as follows; Section II present the essential preliminaries as notation and the quaternion framework, while in Section III the problem formulation is presented along with essential sensor models. In Section IV the filter is derived, including implementation notes, while Section V presentes the quadrotor model derivation. Results from simulation are presented in Section VI while a discussion on future work is given in Section VII.

\section{PRELIMINARIES}

\section{A. Notation and reference frames}

Throughout this paper scalar values are denoted in normal face, vectors in boldface while matrices are written in capital boldface letters. The time derivative is denoted as $\dot{\mathbf{x}}=\frac{d \mathbf{x}}{d t}$, the Euclidean norm is denoted by $\|\cdot\|$ and any estimated value will be denoted by a hat, $(\cdot)$. Note that $\mathbf{I}_{n \times n}$ denotes an $n \times n$ identity matrix while $\mathbf{0}_{n \times m}$ denotes a $n \times m$ matrix of zeros. Vectors are decomposed in different reference frames denoted by superscripts, where $\mathcal{F}^{b}$ is the body frame, $\mathcal{F}^{t p p}$ is the propeller tip-path-plane and $\mathcal{F}^{n}$ is the North-East-Down (NED) frame which is assumed to be inertial. The rotation matrix from $\mathcal{F}^{b}$ to $\mathcal{F}^{n}$ is denoted as $\mathbf{R}_{b}^{n} \in S O(3)$, where

$$
S O(3)=\left\{\mathbf{R} \in \mathbb{R}^{3 \times 3}: \mathbf{R}^{\top} \mathbf{R}=\mathbf{I}_{3 \times 3}, \operatorname{det}(\mathbf{R})=1\right\}
$$

is the special orthogonal group of order three. In this work we use quaternions to parametrize $S O(3)$, and the equivalent attitude quaternion from $\mathcal{F}^{b}$ to $\mathcal{F}^{n}$ will be denoted as $\mathbf{q}_{n, b}$. The angular velocity is denoted $\boldsymbol{\omega}_{b, c}^{a}$, which is to say the angular velocity of $\mathcal{F}^{c}$ relative $\mathcal{F}^{b}$ referenced in $\mathcal{F}^{a}$. For any arbitrary vectors $\mathbf{v}_{1}, \mathbf{v}_{2} \in \mathbb{R}^{3}$, we denote the cross-product operator as $\mathbf{S}\left(\mathbf{v}_{1}\right) \mathbf{v}_{2}=\mathbf{v}_{1} \times \mathbf{v}_{2}$.

\section{B. Attitude parametrization}

The attitude of a rigid-body is represented by a $3 \times 3$ orthogonal matrix with unity determinant, i.e. the rotation matrix, belonging to the special orthogonal group $S O(3)$ [17]. The orthogonality of the rotation matrix and the fact that it consists of nine elements makes the rotation matrix unsuitable for filter applications. The lowest dimensional global non-singular representation of $S O(3)$ possible is the unit quaternion [17]. A quaternion can be defined as a complex number with one real part $\eta$ and three imaginary parts $\epsilon=\left[\epsilon_{1} \epsilon_{2} \epsilon_{3}\right]^{\top}$ [18], and if the quaternion satisfy the unity norm constraint it belongs to the set of unit quaternions

$$
\mathbb{H}=\left\{\mathbf{q} \in \mathbb{R}^{4}: \mathbf{q}=\left[\eta \boldsymbol{\epsilon}^{\top}\right]^{\top}, \eta \in \mathbb{R}, \boldsymbol{\epsilon} \in \mathbb{R}^{3},\|\mathbf{q}\|=\mathbf{1}\right\} .
$$

Adhering to the Hamilton quaternion convention and its associated algebra, described in [19], the product of two quaternions, $\mathbf{q}=\left[\eta_{q} \boldsymbol{\epsilon}_{q}^{\top}\right]^{\top}$ and $\mathbf{p}=\left[\eta_{p} \boldsymbol{\epsilon}_{p}^{\top}\right]^{\top}$, is calculated as

$$
\mathbf{p} \otimes \mathbf{q}=\mathbf{T}(\mathbf{p}) \mathbf{q}=\left[\begin{array}{c}
\eta_{p} \eta_{q}-\boldsymbol{\epsilon}_{p}^{\top} \boldsymbol{\epsilon}_{q} \\
\eta_{p} \boldsymbol{\epsilon}_{q}+\eta_{q} \boldsymbol{\epsilon}_{p}+\boldsymbol{\epsilon}_{p} \times \boldsymbol{\epsilon}_{q}
\end{array}\right]
$$

where

$$
\mathbf{T}(\mathbf{p})=\left[\begin{array}{cc}
\eta_{p} & -\boldsymbol{\epsilon}_{p}^{\top} \\
\boldsymbol{\epsilon}_{p} & \eta_{p} \mathbf{I}_{3 x 3}+\mathbf{S}\left(\boldsymbol{\epsilon}_{p}\right)
\end{array}\right] .
$$

The quaternion norm is defined by

$$
\|\mathbf{q}\|=\sqrt{\mathbf{q} \otimes \mathbf{q}^{*}}=\sqrt{\eta^{2}+\epsilon_{1}^{2}+\epsilon_{2}^{2}+\epsilon_{3}^{2}}
$$

where the quaternion conjugate is

$$
\mathbf{q}^{*}=\left[\begin{array}{c}
\eta \\
-\boldsymbol{\epsilon}
\end{array}\right]
$$

If $\mathbf{p}$ and $\mathbf{q}$ both meet the unity-norm constraint, the quaternion resulting from their composition will also be unitynorm. Finally the kinematics of the attitude quaternion can be defined, in the case of one frame moving relative to another, as

$$
\dot{\mathbf{q}}_{n, b}(t)=\frac{1}{2} \mathbf{q}_{n, b}(t) \otimes \boldsymbol{\omega}_{n, b}^{b}(t)
$$

where $\boldsymbol{\omega}_{n, b}^{b}(t) \in \mathbb{R}^{4}$ is the angular velocity, represented here as a pure quaternion, i.e. a quaternion with zero real part.

\section{PROBLEM FORMULATION}

The attitude estimation problem is to determine the orientation of a moving frame with respect to the inertial reference frame. More precisely, suppose that at time $t$ a set of $n(t) \in \mathbb{N}$ vector measurements is available, defined by the relation

$$
\mathbf{v}_{m i}^{b}(t)=\mathbf{q}_{n, b}^{*}(t) \otimes \mathbf{v}_{m i}^{n}(t) \otimes \mathbf{q}_{n, b}(t)+\boldsymbol{v}_{i}(t), i=1, \ldots, n(t)
$$

where $\mathbf{v}_{m i}^{b}(t) \in \mathbb{R}^{3}$ is the vector measurements in $\mathcal{F}^{b}$, $\mathbf{v}_{m i}^{n}(t) \in \mathbb{R}^{3}$ is the related known reference vectors in $\mathcal{F}^{n}$, and $\boldsymbol{v}_{i}(t)$ is the measurement errors. Further consider the attitude kinematics in (7) and that measurements of the angular velocity of the body frame, $\boldsymbol{\omega}_{m}^{b}(t) \in \mathbb{R}^{3}$, is available. Our task is to obtain an optimal estimate, $\hat{\mathbf{q}}_{n, b}(t)$, of the vehicles attitude at any time $t$ given vector and gyro measurements, subject to unmodeled errors and disturbances.

\section{A. Inertial sensors}

The available gyroscope has the associated standard error model [20], stated as

$$
\begin{aligned}
\boldsymbol{\omega}_{m}^{b}(t) & =\boldsymbol{\omega}_{n, b}^{b}(t)+\boldsymbol{\beta}_{g}(t)+\mathbf{w}_{g}(t) \\
\dot{\boldsymbol{\beta}}_{g}(t) & =\mathbf{w}_{b}(t)
\end{aligned}
$$

where $\boldsymbol{\beta}_{g}(t) \in \mathbb{R}^{3}$ is the gyroscope bias, $\mathbf{w}_{g}(t) \in \mathbb{R}^{3}$ is the gyroscope measurement noise, and $\mathbf{w}_{b}(t) \in \mathbb{R}^{3}$ is a 
Gaussian white noise process with zero-mean and variance $\sigma_{w}^{2}$, modeling the bias as a slowly time-varying disturbance. Defining the combined gyroscope noise

$$
\mathbf{w}(t)=\left[\begin{array}{l}
\mathbf{w}_{g}(t) \\
\mathbf{w}_{b}(t)
\end{array}\right]
$$

with associated autocovariance

$$
\mathbf{Q}(t)=E\left\{\mathbf{w}(t) \mathbf{w}(t)^{\top}\right\} \approx \sigma_{w}^{2} \mathbf{I}_{6 \times 6}
$$

where $E\{\cdot\}$ denotes the expectation value. Two vector measurements is provided by a standard IMU; i.e. measurements from accelerometer and magnetometer. The latter measure the inertial magnetic field expressed in body frame

$$
\mathbf{v}_{m 1}^{b}(t)=\mathbf{q}_{n, b}^{*}(t) \otimes \mathbf{v}_{m 1}^{n}(t) \otimes \mathbf{q}_{n, b}(t)+\boldsymbol{v}_{1}(t)
$$

where $\mathbf{v}_{m 1}^{n}(t) \in \mathbb{R}^{3}$ represent the Earth's magnetic field magnitude and direction, and $\boldsymbol{v}_{1}(t) \in \mathbb{R}^{3}$ is the magnetometer measurement noise. We assume that the magnetometer is well calibrated in terms of hard and soft iron effects prior to flight and that the IMU is mounted in the center of mass of the vehicle. The accelerometer measure spesific force, i.e.

$$
\mathbf{v}_{m 2}^{b}(t)=\dot{\mathbf{v}}^{b}(t)-\mathbf{f}_{g}^{b}(t)+\boldsymbol{v}_{2}(t)
$$

where $\dot{\mathbf{v}}^{b}(t) \in \mathbb{R}^{3}$ is the change in body velocity, $\mathbf{f}_{g}^{b}(t) \in$ $\mathbb{R}^{3}$ is the gravity vector in $\mathcal{F}^{b}$, and $\boldsymbol{v}_{2}(t) \in \mathbb{R}^{3}$ is the accelerometer measurement noise. Here we make the assumption of weak acceleration mentioned previously, and the measurement equation then becomes

$$
\mathbf{v}_{m 2}^{b}(t)=-\mathbf{q}_{n, b}^{*}(t) \otimes \mathbf{v}_{m 2}^{n}(t) \otimes \mathbf{q}_{n, b}(t)+\boldsymbol{v}_{2}(t)
$$

where $\mathbf{v}_{m 2}^{n}(t)=\mathbf{f}_{g}^{n}(t)=\left[\begin{array}{lll}0 & 0 & g\end{array}\right]^{\top}$ with $g$ being the gravity constant. Both magnetometer and accelerometer measurement noise can be gathered into one vector as

$$
\boldsymbol{v}(t)=\left[\begin{array}{l}
\boldsymbol{v}_{1} \\
\boldsymbol{v}_{2}
\end{array}\right]
$$

which is assumed to be Gaussian white noise with zero-mean and variance $\sigma_{v}^{2}$, where $\sigma_{v}^{2}=\left[\begin{array}{ll}\sigma_{m 1}^{2} & \sigma_{m 2}^{2}\end{array}\right]^{\top}$. The assosiated autocovariance is given as

$$
\mathbf{R}(t)=E\left\{\boldsymbol{v}(t) \boldsymbol{v}(t)^{\top}\right\} \approx \sigma_{v}^{2} \mathbf{I}_{6 \times 6} .
$$

All time-dependence of variables is, for brevity, not stated explicitly from here on.

\section{Multiplicative EKF}

When designing a quaternion based EKF special care must be taken with regards to the unit constraint on the quaternion, since the construction may lead to a potential singular covariance matrix and an unstable filter. The MEKF approach for dealing with this problem is based on reducing the dimension of the attitude representation so that three parameters are used instead of four to describe the covariance of the quaternion. The true attitude in the MEKF formalism is represented as the quaternion product

$$
\overline{\mathbf{q}}=\hat{\mathbf{q}} \otimes \delta \mathbf{q}(\delta \boldsymbol{\epsilon})
$$

where $\hat{\mathbf{q}} \in \mathbb{H}$ is the estimated quaternion and $\delta \mathbf{q}(\delta \boldsymbol{\epsilon}) \in \mathbb{H}$ is a quaternion representing the rotation from $\hat{\mathbf{q}}$ to the true attitude $\overline{\mathbf{q}} \in \mathbb{H}$. The MEKF maintains the attitude error $\delta \boldsymbol{\epsilon} \in \mathbb{R}^{3}$, instead of the attitude, in the state estimate and covariance. The attitude error never approaches a singularity since it only represents small attitude errors and can be constructed as

$$
\delta \mathbf{q}(\delta \boldsymbol{\epsilon})=\left[\begin{array}{c}
\sqrt{1-\delta \boldsymbol{\epsilon}^{\top} \delta \boldsymbol{\epsilon}} \\
\delta \boldsymbol{\epsilon}
\end{array}\right]
$$

\section{A. Filter structure}

A general model of a nonlinear system is set up using the common state propagation and measurement equations as

$$
\begin{aligned}
\dot{\mathbf{x}} & =\mathbf{f}(\mathbf{x}, \mathbf{u})+\Gamma \mathbf{w} \\
\mathbf{y} & =\mathbf{h}(\mathbf{x})+\boldsymbol{v}
\end{aligned}
$$

where $\mathbf{u}$ is the input vector, $\boldsymbol{\Gamma}$ is the cross-correlation matrix, while $\mathbf{w}$ and $\boldsymbol{v}$ is the process and measurement noise respectively. Standard MEKF procedure is divided into three parts namely; measurements update, reset and time update -cf. [21]. The measurement update is summarized as

$$
\begin{aligned}
\mathbf{K} & =\mathbf{P}^{-} \mathbf{H}^{\top}\left[\mathbf{H} \mathbf{P}^{-} \mathbf{H}^{\top}+\mathbf{R}\right]^{-1} \\
\hat{\mathbf{x}}^{+} & =\hat{\mathbf{x}}^{-}+\mathbf{K}\left[\mathbf{y}-\mathbf{h}\left(\hat{\mathbf{x}}^{-}\right)\right] \\
\mathbf{P}^{+} & =(\mathbf{I}-\mathbf{K H}) \mathbf{P}^{-}(\mathbf{I}-\mathbf{K H})^{\top}+\mathbf{K} \mathbf{R} \mathbf{K}^{\top}
\end{aligned}
$$

where $\hat{\mathbf{x}}^{-}, \hat{\mathbf{x}}^{+} \in \mathbb{R}^{m}$ is the predicted and corrected state estimate, containing the attitude error $\delta \boldsymbol{\epsilon}$. The matrix $\mathbf{K} \in$ $\mathbb{R}^{m \times 3 n}$ is the Kalman gain, $\mathbf{P}^{-}, \mathbf{P}^{+} \in \mathbb{R}^{m \times m}$ are the predicted and corrected error covariance, and $\mathbf{H} \in \mathbb{R}^{3 \times 3 n}$ is the Jacobian of the measurement equation $\mathbf{h}(\cdot) \in \mathbb{R}^{3 n}$. The reset operation eliminates the need to propagate two representations of attitude by moving the attitude information from $\delta \mathbf{q}(\delta \hat{\boldsymbol{\epsilon}})$ to $\hat{\mathbf{q}}$,

$$
\begin{aligned}
\hat{\mathbf{q}}^{-} & =\hat{\mathbf{q}}^{+} \otimes \delta \mathbf{q}(\hat{\delta \boldsymbol{\epsilon}}) \\
\hat{\delta \boldsymbol{\epsilon}} & =\left[\begin{array}{lll}
0 & 0 & 0
\end{array}\right]^{\top}
\end{aligned}
$$

Finally the time update is performed as

$$
\begin{aligned}
\hat{\mathbf{x}}^{-} & =\mathbf{f}\left(\hat{\mathbf{x}}^{+}, \mathbf{u}\right) \\
\mathbf{P}^{-} & =\mathbf{F} \mathbf{P}^{+} \mathbf{F}^{\top}+\boldsymbol{\Gamma Q Q} \Gamma^{\top}
\end{aligned}
$$

where $\mathbf{F} \in \mathbb{R}^{m \times m}$ is the state transition matrix, i.e. the Jacobian of the propagation equation $\mathbf{f}(\cdot) \in \mathbb{R}^{m}$.

The model states are choosen as the vehicle attitude and gyroscope bias

$$
\mathbf{x}=\left[\mathbf{q}_{n, b}^{\top} \boldsymbol{\beta}^{\top}\right]^{\top}
$$

and the model inputs given as the gyroscope measurements $\mathbf{u}=\boldsymbol{\omega}_{m}^{b}$. Deriving the filter we first introduce the nonlinear equations associated with the true state of our system, $\overline{\mathbf{x}}=$ $\left[\overline{\mathbf{q}}_{n, b}^{\top} \overline{\boldsymbol{\beta}}^{\top}\right]^{\top}$. For simplicity we set $\overline{\boldsymbol{\omega}}=\mathbf{u}-\overline{\boldsymbol{\beta}}$, and obtain the nonlinear equations

$$
\dot{\overline{\mathbf{x}}}=\left[\begin{array}{c}
\dot{\overline{\mathbf{q}}}_{n, b}^{\top} \\
\dot{\overline{\boldsymbol{\beta}}}
\end{array}\right]=\left[\begin{array}{c}
\frac{1}{2} \overline{\mathbf{q}} \otimes \overline{\boldsymbol{\omega}} \\
\mathbf{0}
\end{array}\right] .
$$


As mentioned previously the MEKF filter state contains the attitude error not the attitude itself, so the state is defined as

$$
\mathbf{x}=\left[\delta \boldsymbol{\epsilon}^{\top} \boldsymbol{\beta}^{\top}\right]^{\top}
$$

The time-derivative of the attitude error is found by first rearranging and then differentiating equation (17) as

$$
\dot{\delta \mathbf{q}}(\delta \boldsymbol{\epsilon})=\dot{\hat{\mathbf{q}}}^{-1} \otimes \overline{\mathbf{q}}+\hat{\mathbf{q}}^{-1} \otimes \dot{\overline{\mathbf{q}}}
$$

Since we, in the discrete-time filter, do not time propagate $\hat{\mathbf{q}}$ it can be treated as a constant between each sample. This leads to

$$
\begin{aligned}
\dot{\delta \mathbf{q}}(\delta \boldsymbol{\epsilon})=\left[\begin{array}{c}
\dot{\delta \boldsymbol{\eta}} \\
\dot{\delta \boldsymbol{\epsilon}}
\end{array}\right] & =\hat{\mathbf{q}}^{-1} \otimes \dot{\overline{\mathbf{q}}}=\frac{1}{2} \hat{\mathbf{q}}^{-1} \otimes \overline{\mathbf{q}} \otimes \overline{\boldsymbol{\omega}} \\
& =\frac{1}{2} \delta \mathbf{q}(\delta \boldsymbol{\epsilon}) \otimes \overline{\boldsymbol{\omega}} \\
& =\frac{1}{2} \mathbf{T}(\delta \mathbf{q}(\delta \boldsymbol{\epsilon})) \overline{\boldsymbol{\omega}}
\end{aligned}
$$

where $\mathbf{T}(\cdot)$ is given in equation (4). This then gives the differential equation for $\delta \epsilon$ as

$$
\dot{\delta \boldsymbol{\epsilon}}=\frac{1}{2}\left[\mathbf{I}_{3 x 3} \sqrt{1-\delta \boldsymbol{\epsilon}^{\top} \delta \boldsymbol{\epsilon}}+\mathbf{S}(\delta \boldsymbol{\epsilon})\right] \overline{\boldsymbol{\omega}} .
$$

Discretizing the systems nonlinear differential equations one obtains

$$
\begin{aligned}
\mathbf{x}_{k+1} & =\mathbf{f}_{k}\left(\mathbf{x}_{k}, \mathbf{u}_{k}\right)+h \boldsymbol{\Gamma}_{k} \mathbf{w}_{k} \\
\mathbf{y}_{k} & =\mathbf{h}_{k}\left(\mathbf{x}_{k}\right)+\boldsymbol{v}_{k}
\end{aligned}
$$

where $h \in \mathbb{R}_{>0}$ is the time step. By Euler's discretizing method and in terms of the filter state this becomes

$$
\mathbf{f}_{k}\left(\hat{\mathbf{x}}_{k}, \mathbf{u}_{k}\right)=\hat{\mathbf{x}}_{k}+h\left[\begin{array}{c}
\frac{1}{2}[\mathbf{u}-\hat{\boldsymbol{\beta}}] \\
\mathbf{0}_{3 x 1}
\end{array}\right]
$$

and the Jacobian is found to be

$$
\mathbf{F}_{k}=\left.\frac{\partial \mathbf{f}_{k}}{\partial \mathbf{x}_{k}}\right|_{\mathbf{x}_{k}=\hat{\mathbf{x}}_{k}}=\mathbf{I}_{6 x 6}+h\left[\begin{array}{cc}
-\frac{1}{2} \mathbf{S}(\mathbf{u}-\hat{\boldsymbol{\beta}}) & -\frac{1}{2} \mathbf{I}_{3 x 3} \\
\mathbf{0}_{3 x 3} & \mathbf{0}_{3 x 3}
\end{array}\right] .
$$

Further we define the measurement equations as

$$
\mathbf{y}=\left[\begin{array}{c}
\mathbf{v}_{m 1}^{b} \\
\mathbf{v}_{m 2}^{b}
\end{array}\right]=\left[\begin{array}{l}
\delta \mathbf{q}_{\hat{b}, b}^{*} \otimes \hat{\mathbf{q}}_{n, \hat{b}}^{*} \otimes \mathbf{v}_{m 1}^{n} \otimes \hat{\mathbf{q}}_{n, \hat{b}} \otimes \delta \mathbf{q}_{\hat{b}, b} \\
\delta \mathbf{q}_{\hat{b}, b}^{*} \otimes \hat{\mathbf{q}}_{n, \hat{b}}^{*} \otimes \mathbf{v}_{m 2}^{n} \otimes \hat{\mathbf{q}}_{n, \hat{b}} \otimes \delta \mathbf{q}_{\hat{b}, b}
\end{array}\right] .
$$

To derive the Jacobian for the measurement equations we again use the fact that $\hat{\mathbf{q}}$ does not change during time propagation. By defining the intermediate vector $\mathbf{v}_{m}^{\hat{b}}$ in the intermediate frame $\mathcal{F}^{\hat{b}}$ as

$$
\mathbf{v}_{m i}^{\hat{b}}=\hat{\mathbf{q}}_{n, \hat{b}}^{*} \otimes \mathbf{v}_{m i}^{n} \otimes \hat{\mathbf{q}}_{n, \hat{b}} \quad i=1,2
$$

and expanding the terms in (32) by using (3) we obtain an auxiliary derivative function by

$$
\begin{aligned}
& \mathbf{V}\left(\delta \boldsymbol{\epsilon}, \mathbf{v}_{m i}^{\hat{b}}\right)=\frac{\partial}{\partial \delta \boldsymbol{\epsilon}}\left\{\delta \mathbf{q}(\delta \boldsymbol{\epsilon})^{*} \otimes \mathbf{v}_{m i}^{\hat{b}} \otimes \delta \mathbf{q}(\delta \boldsymbol{\epsilon})\right\} \\
& =\frac{\partial}{\partial \delta \boldsymbol{\epsilon}}\left\{\left[\left(\eta^{2}-\delta \boldsymbol{\epsilon}^{\top} \delta \boldsymbol{\epsilon}\right) \mathbf{I}_{3 \times 3}+2 \delta \boldsymbol{\epsilon} \delta \boldsymbol{\epsilon}^{\top}-2 \eta S(\delta \boldsymbol{\epsilon})\right] \mathbf{v}_{m i}^{\hat{b}}\right\}
\end{aligned}
$$

After differentiating and rearranging the terms one can find

$$
\begin{aligned}
\mathbf{V}\left(\delta \boldsymbol{\epsilon}, \mathbf{v}_{m i}^{\hat{b}}\right)= & 2 \sqrt{1-\delta \boldsymbol{\epsilon}^{\top} \delta \boldsymbol{\epsilon}} \mathbf{S}\left(\mathbf{v}_{m i}^{\hat{b}}\right) \\
& -\frac{2}{\sqrt{1-\delta \boldsymbol{\epsilon}^{\top} \delta \boldsymbol{\epsilon}}} \mathbf{S}\left(\mathbf{v}_{m i}^{\hat{b}}\right) \delta \boldsymbol{\epsilon} \delta \boldsymbol{\epsilon}^{\top} \\
& +2 \mathbf{v}_{m i}^{\hat{b}^{\top} \delta \boldsymbol{\epsilon} \mathbf{I}_{3 x 3}}+2 \delta \boldsymbol{\epsilon} \mathbf{v}_{m i}^{\hat{b} \top}-4 \mathbf{v}_{m i}^{\hat{b}} \delta \boldsymbol{\epsilon}^{\top}
\end{aligned}
$$

which then can be used to define the Jacobian of the measurement equation as

$$
\mathbf{H}_{k}=\left.\frac{\partial \mathbf{h}_{k}}{\partial \mathbf{x}_{k}}\right|_{\mathbf{x}_{k}=\hat{\mathbf{x}}_{k}}=\left[\begin{array}{ll}
\mathbf{V}\left(\delta \boldsymbol{\epsilon}, \mathbf{v}_{m 1}^{\hat{b}}\right) & \mathbf{0}_{3 x 3} \\
\mathbf{V}\left(\delta \boldsymbol{\epsilon}, \mathbf{v}_{m 2}^{\hat{b}}\right) & \mathbf{0}_{3 x 3}
\end{array}\right]
$$

\section{B. Adaptive fading EKF}

The adaptive fading EKF has been derived in [9]. The innovation covariance is calculated as

$$
\mathbf{C}_{k}=E\left[\boldsymbol{\zeta}_{k} \boldsymbol{\zeta}_{k}^{\top}\right]=\mathbf{H}_{k} \mathbf{P}_{k}^{-} \mathbf{H}_{k}^{\top}+\mathbf{R}_{k}
$$

with $\boldsymbol{\zeta}_{k}=\mathbf{y}_{k}-\mathbf{h}_{k}\left(\hat{\mathbf{x}}_{k}^{-}\right)$being the innovation. The innovation of the filter is affected by unaccounted errors such as unknown fault bias, unmodeled dynamics and even unknown initial condition. It is shown in [9] that any unaccounted errors are directly involved in the computation of the innovation, this means that the change of innovation covariance can be used for an adaptive filter. The increased innovation covariance can be estimated as

$$
\hat{\mathbf{C}}_{k}=\frac{1}{M-1} \sum_{i=k-M+1}^{k} \boldsymbol{\zeta}_{i} \boldsymbol{\zeta}_{i}^{\top}
$$

where $M \in \mathbb{N}$ is a window size. The relation between $\mathbf{C}_{k}$ and $\hat{\mathbf{C}}_{k}$ is defined as $\hat{\mathbf{C}}_{k}=\alpha_{k} \mathbf{C}_{k}$, where $\alpha_{k} \in \mathbb{R}$ is used to make the filter adaptive. In accelerated flight the assumption of weak acceleration does not hold, resulting in corrupt measurements with regards to the measurement equations and an increased innovation covariance. This lack of information can be compensated by decreasing the Kalman gain and thus relying less on the measurement information. The standard measurement update in (20) can then be altered by redefining the Kalman gain calculation as

$$
K_{k}=\frac{1}{\alpha_{k}} \mathbf{P}_{k} \mathbf{H}_{k}\left[\mathbf{H}_{k} \mathbf{P}_{k} \mathbf{H}_{k}^{\top}+\mathbf{R}_{k}\right]^{-1} .
$$

Calculating the innovation is done during the time update (22), after the state is propagated ahead, given as

$$
\begin{aligned}
\boldsymbol{\zeta}_{k} & =\mathbf{y}_{k}-\mathbf{h}_{k}\left(\hat{\mathbf{x}}_{k}\right) \\
\hat{\mathbf{C}}_{k} & =\frac{1}{M-1} \sum_{i=k-M+1}^{k} \zeta_{i} \zeta_{i}^{\top} \\
\alpha_{k} & =\max \left\{1, \frac{\operatorname{tr}\left(\overline{\mathbf{C}}_{\mathrm{k}}\right)}{\operatorname{tr}\left(\mathbf{C}_{\mathrm{k}}\right)}\right\}
\end{aligned}
$$

where $\operatorname{tr}(\cdot) \in \mathbb{R}$ indicate the trace function and $\max \{\cdot\} \in \mathbb{R}$ is the function returning the largest value. 


\section{APPLICATION TO THE QUADROTOR PLATFORM}

\section{A. Quadrotor modelling}

Extensive derivation of a quadrotor nonlinear dynamic model can be found in [22], [23], [24]. In the following, we briefly present the essential rigid-body equations in addition to some of the dominant aerodynamic forces and how these affect the sensor measurements. The $\mathcal{F}^{n}$ frame is assumed inertial and the origin of $\mathcal{F}^{b}$ coincide with the center of mass of the quadrotor. The quadrotor rigid-body equations of motion are

$$
\begin{gathered}
\dot{\mathbf{p}}_{n, b}^{n}=\mathbf{q}_{n, b} \otimes \mathbf{v}^{b} \otimes \mathbf{q}_{n, b}^{*} \\
\dot{\mathbf{v}}^{b}=\mathbf{f}_{g}^{b}-\mathbf{S}\left(\boldsymbol{\omega}_{n, b}^{b}\right) \mathbf{v}^{b}-\frac{1}{m} \mathbf{q}_{b, t p p} \otimes \mathbf{f}_{t}^{t p p} \otimes \mathbf{q}_{b, t p p}^{*}-\frac{1}{m} \mathbf{f}_{d}^{b} \\
\dot{\mathbf{q}}_{n, b}=\frac{1}{2} \mathbf{q}_{n, b} \otimes \boldsymbol{\omega}_{n, b}^{b} \\
\mathbf{J} \dot{\boldsymbol{\omega}}_{n, b}^{b}=-\mathbf{S}\left(\boldsymbol{\omega}_{n, b}^{b}\right) \mathbf{J} \boldsymbol{\omega}_{n, b}^{b}+\mathbf{g}_{a}^{b}+\boldsymbol{\tau}^{b}+\boldsymbol{\tau}_{d}^{b}
\end{gathered}
$$

where $\dot{\mathbf{p}}_{n, b}^{n} \in \mathbb{R}^{3}$ is the position vector from the origin of $\mathcal{F}^{n}$ to the origin of $\mathcal{F}^{b}, \mathbf{v}^{b} \in \mathbb{R}^{3}$ is the velocity vector in $\mathcal{F}^{b}$, $m \in \mathbb{R}$ is the quadrotors mass, $\mathbf{f}_{g}^{b}=\mathbf{R}_{n}^{b} \mathbf{f}_{g}^{n}$ where $\mathbf{f}_{g}^{n} \in \mathbb{R}^{3}$ is the inertial gravitational force, and $\mathbf{J} \in \mathbb{R}^{3 \times 3}$ is the inertia matrix. The vector $\mathbf{f}_{t}^{t p p} \in \mathbb{R}^{3}$ is the tip-path-plane thrust vector, i.e. the thrust vector that only has a component in the z-direction. If the quadrotor is stationary and no wind is present $\mathcal{F}^{t p p}$ coincides with $\mathcal{F}^{b}$. The vector can be calculated as

$$
\mathbf{f}_{t}^{t p p}=\left[\begin{array}{c}
0 \\
0 \\
C_{T}\left(\varpi_{1}^{2}+\varpi_{2}^{2}+\varpi_{3}^{2}+\varpi_{4}^{2}\right)
\end{array}\right]
$$

where $C_{T} \in \mathbb{R}$ is the thrust coefficient which can be determined through static thrust tests [22] and $\varpi_{i} \in \mathbb{R}$, $i=1,2,3,4$, is the rotational velocity of the i'th rotor. Due to blade flapping, a phenomenon thoroughly described in helicopter literature - $c f$. [25], [26], the thrust vector experiences a deflection from the body z-axis resulting in forces in the body horizontal plane. The body frame moments are also related to the rotor speeds of the motors and are calculated as

$$
\boldsymbol{\tau}^{b}=\left[\begin{array}{c}
C_{T} l\left(\varpi_{4}-\varpi_{2}\right) \\
C_{T} l\left(\varpi_{1}-\varpi_{3}\right) \\
C_{Q}\left(\varpi_{1}-\varpi_{2}+\varpi_{3}-\varpi_{4}\right)
\end{array}\right]
$$

where $C_{Q} \in \mathbb{R}$ is the motor parameter relating the angular velocity of the motor to the rotor torque and $l \in \mathbb{R}$ is the arm length of the quadrotor. The vector $\mathbf{g}_{a}^{b} \in \mathbb{R}^{3}$ is the gyroscopic moment of the rotors on the airframe, calculated as [27]

$$
\mathbf{g}_{a}^{b}=-\sum_{i=1}^{4}(-1)^{i+1} \mathbf{S}\left(\boldsymbol{\omega}_{n, b}^{b}\right) \mathbf{J}_{p} \varpi_{i}
$$

where $\mathbf{J}_{p} \in \mathbb{R}^{3 \times 3}$ is the moment of inertia of a rotor around its axis and $\varpi_{i}=\left[\begin{array}{ccc}0 & 0 & \varpi_{i}\end{array}\right]^{\top}$. Finally we have the drag forces associated with quadrotor flight, $\mathbf{f}_{d}^{b} \in \mathbb{R}^{3}$, including, among others, the rotor relative momentum drag and the body relative parasitic drag $-c f$. [22]. The rotor relative drag forces generate drag torques, $\tau_{d}^{b} \in \mathbb{R}^{3}$, due to displacement of the rotors.

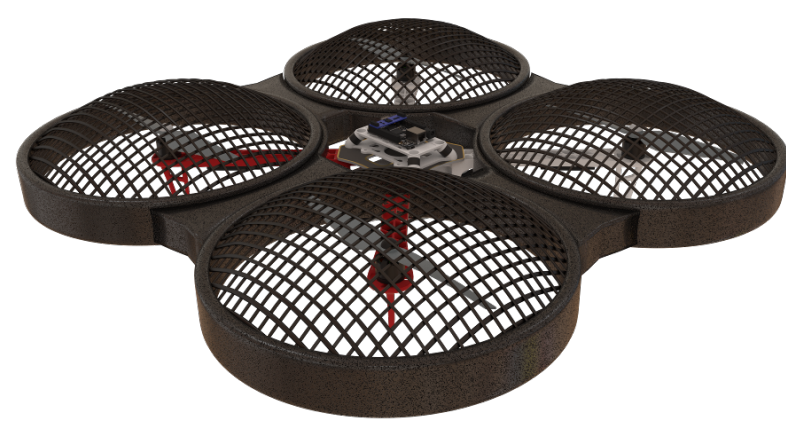

Fig. 1. The UiTRotor platform

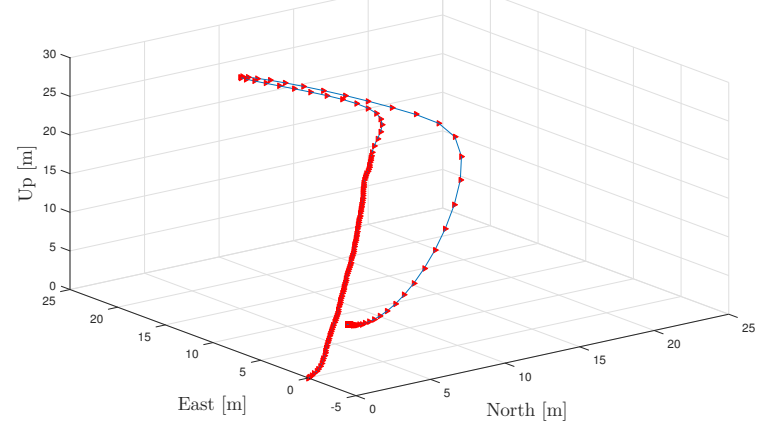

Fig. 2. The simulated trajectory

\section{RESULTS}

\section{A. Simulations}

In order to evaluate the filter performance a simulator was set up in Matlab Simulink using the quadrotor dynamics described in Section V-A and system parameters assosiated with UCAS-LAB's UiTRotor platform, shown in Figure 1. To test how well the filter performed, a trajectory was designed and fed to a nonlinear controller. At first, the quadrotor follows a slowly rising trajectory, it then swithces to track an elevation variating semicircle before finally it maneuvers to a fixed waypoint. The simulated trajectory is shown in Figure 2. The initial attitude of the quadrotor is $\mathbf{q}_{n, b}=\left[\begin{array}{llll}1 & 0 & 0 & 0\end{array}\right]^{\top}$, and the gyroscope bias is set to $\boldsymbol{\beta}=\left[\begin{array}{lll}0.001 & 0.001 & 0.001\end{array}\right]^{\top} \mathrm{deg} / \mathrm{s}$. The filter is initiated with an initial state $\hat{\mathbf{x}}_{0}=\mathbf{0}_{1 \times 6}$, the initial attitude $\hat{\mathbf{q}}_{0}=\frac{1}{\sqrt{4}} \mathbf{I}_{1 \times 4}$, window size $M=10$, and initial error covariance $\mathbf{P}_{0}=$ $\left[\begin{array}{llllll}0.1 & 0.1 & 0.1 & 0.01 & 0.01 & 0.01\end{array}\right]^{\top}$. The two vector measurement have corresponding inertial reference vectors $\mathbf{v}_{m 1}^{n}=$

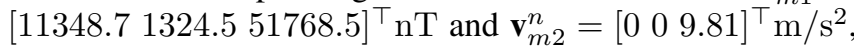
and the measurement noise variance, in (16), has values $\sigma_{m 1}^{2}=4.5 \times 10^{-4} \mathrm{deg}^{2}$ and $\sigma_{m 2}^{2}=1.5 \times 10^{-3} \mathrm{deg}^{2}$.

In order to evaluate the effect of the adaptive stage addition to the MEKF we simulate two attitude estimators, with and without adaptation. The error is quantazied as an error quaternion, calculated as $\mathbf{q}_{e}=\hat{\mathbf{q}} \otimes \overline{\mathbf{q}}^{*}$, and for ease of illustration the scalar part is adapted as $\eta=1-\eta$. In Figure 3 


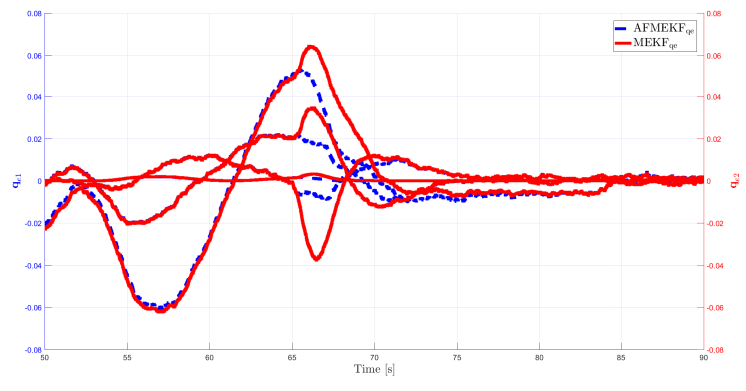

Fig. 4. Error quaternions aggressive maneuver

the error quaternion for the two filters is presented along with the innovation measure $\alpha$ associated with the AFMEKF. The interesting part is after about 66 seconds when the quadrotor switches to the fixed waypoint, resulting in an aggressive maneuver. Clearly the innovation increases and as a result the AFMEKF performs better than the non-adaptive MEKF.
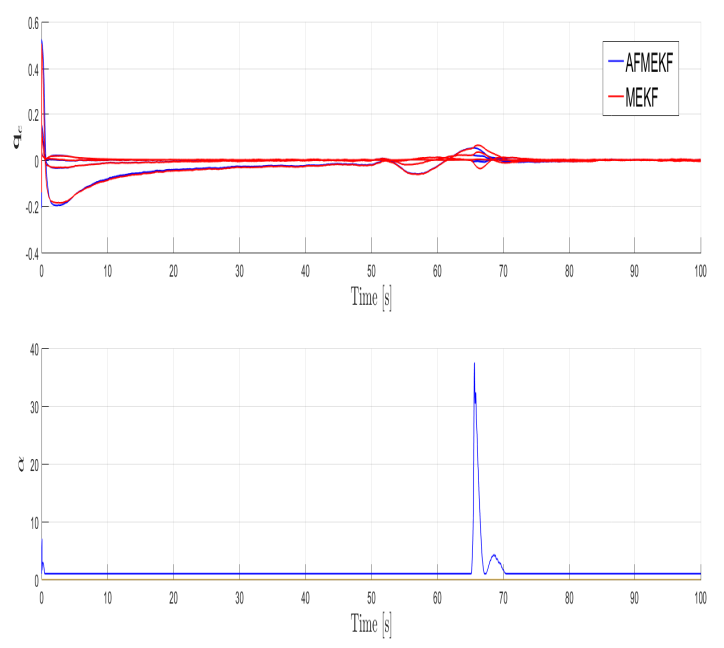

Fig. 3. Upper: Error quaternion AFMEKF and MEKF. Lower: Innovation measure

A closer look of the error quaternions during that aggressive maneuver is presented in Figure 4.

\section{CONCLUSION AND FUtURE WORK}

An adaptive fading multiplicative extended Kalman filter has been implemented for the task of quadrotor attitude estimation. Simulations show that in the case of large accelerations, when the measured data is incomplete, the AFMEKF has the ability to improve the state estimates. Future work may include adding horizontal body velocity to the filter state, which will improve attitude estimates.

\section{REFERENCES}

[1] B. Siciliano and O. Khatib, eds., Springer Handbook of Robotics. Springer International Publishing, 2 ed., 2016.

[2] R. Mahony, V. Kumar, and P. Corke, "Multirotor aerial vehicles: Modeling, estimation, and control of quadrotor," IEEE Robotics \& Automation Magazine, vol. 19, no. 3, 2012.
[3] J. L. Crassidis, F. L. Markley, and Y. Cheng, "Survey of nonlinear attitude estimation methods," Journal of Guidance, Control, and Dynamics, vol. 30, no. 1, 2007.

[4] R. E. Kalman et al., "A new approach to linear filtering and prediction problems," Journal of Basic Engineering, vol. 82, no. 1, 1960.

[5] G. L. Smith, S. F. Schmidt, and L. A. McGee, "Application of statistical filter theory to the optimal estimation of position and velocity on board a circumlunar vehicle". National Aeronautics and Space Administration, 1962.

[6] J. K. Hall, N. B. Knoebel, and T. W. McLain, "Quaternion attitude estimation for miniature air vehicles using a multiplicative extended Kalman filter," in Position, Location and Navigation Symposium, IEEE, 2008.

[7] M. W. Mueller, M. Hehn, and R. DAndrea, "Covariance correction step for Kalman filtering with an attitude," Journal of Guidance, Control, and Dynamics, 2016.

[8] E. J. Lefferts, F. L. Markley, and M. D. Shuster, "Kalman filtering for spacecraft attitude estimation," Journal of Guidance, Control, and Dynamics, vol. 5, no. 5, 1982.

[9] K. H. Kim, J. G. Lee, and C. G. Park, "Adaptive two-stage extended Kalman filter for a fault-tolerant INS-GPS loosely coupled system," IEEE Transactions on Aerospace and Electronic Systems, vol. 45, no. $1,2009$.

[10] P. Martin and E. Salaün, "The true role of accelerometer feedback in quadrotor control," in Proceedings to the International Conference on Robotics and Automation, IEEE, 2010.

[11] R. C. Leishman and T. W. McLain, "Multiplicative extended Kalman filter for relative rotorcraft navigation," Journal of Aerospace Information Systems, vol. 12, no. 12, 2014.

[12] J. Macdonald, R. Leishman, R. Beard, and T. McLain, "Analysis of an improved IMU-based observer for multirotor helicopters," Journal of Intelligent \& Robotic Systems, vol. 74, no. 3-4, 2014.

[13] R. C. Leishman, J. C. Macdonald, R. W. Beard, and T. W. McLain, "Quadrotors and accelerometers: State estimation with an improved dynamic model," IEEE Control Systems Magazine, vol. 34, no. 1, 2014.

[14] M.-D. Hua, "Attitude estimation for accelerated vehicles using GPS/INS measurements," Control Engineering Practice, vol. 18, no. 7, 2010.

[15] M.-D. Hua, G. Ducard, T. Hamel, and R. Mahony, "Introduction to nonlinear attitude estimation for aerial robotic systems," Aerospace $L a b, 2014$.

[16] Y. M. Al-Rawashdeh, M. Elshafei, and S. El-Ferik, "Passive attitude estimation using gyroscopes and all-accelerometer IMU" in Proceedings to the 7th International Conference on Mechanical and Aerospace Engineering, 2016.

[17] F. L. Markley, "Attitude estimation or quaternion estimation?," Journal of Astronautical Sciences, vol. 52, no. 1, 2004.

[18] T. I. Fossen, Handbook of marine craft hydrodynamics and motion control. John Wiley \& Sons, 2011.

[19] J. Sola, "Quaternion kinematics for the error-state KF" Laboratoire d'Analyse et d'Architecture des Systemes-Centre national de la recherche scientifique (LAAS-CNRS), Toulouse, France, Tech. Rep, 2012.

[20] M. Park, Error analysis and stochastic modeling of MEMS-based inertial sensors for land vehicle navigation applications, 2004.

[21] F. L. Markley, "Attitude error representations for Kalman filtering," Journal of Guidance, Control, and Dynamics, vol. 26, no. 2, 2003.

[22] M. Bangura, R. Mahony, et al., "Nonlinear dynamic modeling for high performance control of a quadrotor," in Proceedings to the Australasian conference on robotics and automation, 2012.

[23] G. M. Hoffmann, Autonomy for sensor-rich vehicles: Interaction between sensing and control actions. ProQuest, 2008.

[24] P. Pounds, R. Mahony, and P. Corke, "Modelling and control of a large quadrotor robot," Control Engineering Practice, vol. 18, no. 7, 2010.

[25] G. J. Leishman, Principles of helicopter aerodynamics. Cambridge university press, 2006.

[26] R. W. Prouty, Helicopter performance, stability, and control. 1995

[27] T. Hamel, R. Mahony, R. Lozano, and J. Ostrowski, "Dynamic modelling and configuration stabilization for an X4-flyer.," IFAC Proceedings Volumes, vol. 35, no. 1, 2002. 